\title{
Nuclear expression of the RNA-binding protein RBM3 is associated with an improved clinical outcome in breast cancer
}

\author{
Annika Jögi ${ }^{1,2}$, Donal J Brennan ${ }^{3}$, Lisa Rydén ${ }^{4}$, Kristina Magnusson ${ }^{5}$, Mårten Fernö ${ }^{6}$, \\ Olle Stål ${ }^{7}$, Signe Borgquist ${ }^{6}$, Mathias Uhlen ${ }^{8}$, Göran Landberg ${ }^{1}$, Sven Påhlman ${ }^{1,2}$, \\ Fredrik Pontén ${ }^{4}$ and Karin Jirström ${ }^{1,2}$
}

\begin{abstract}
${ }^{1}$ Department of Laboratory Medicine, Center for Molecular Pathology, Malmö University Hospital, Lund University, Malmö, Sweden; ${ }^{2}$ CREATE Health Center for Translational Cancer Research, Lund University, Lund, Sweden; ${ }^{3}$ UCD School of Biomolecular and Biomedical Science, UCD Conway Institute, University College Dublin, Belfield, Dublin, Ireland; ${ }^{4}$ Division of Surgery, Department of Clinical Sciences, Lund University, Lund, Sweden; ${ }^{5}$ Department of Genetics and Pathology, Rudbeck Laboratory, Uppsala University, Uppsala, Sweden; ${ }^{6}$ Division of Oncology, Department of Clinical Sciences, Lund University Hospital, Lund, Sweden; ${ }^{7}$ Department of Biomedicine and Surgery, Division of Oncology, Faculty of Health Sciences, Linköping University, Linköping, Sweden and ${ }^{8}$ Department of Biotechnology, AlbaNova University Center, Royal Institute of Technology, Stockholm, Sweden
\end{abstract}

\begin{abstract}
Single-strand RNA-binding proteins (RBPs) are involved in many aspects of RNA metabolism and in the regulation of gene transcription. The RBP RBM3 was recently suggested to be a proto-oncogene in colorectal cancer; however, such a role has not been corroborated by previous studies in the colon or other tumor types, and the prognostic implications of tumor-specific RBM3 expression remain unclear. Mono-specific antibodies against RBM3 were generated. Antibody specificity was confirmed using siRNA gene silencing, western blotting and immunohistochemistry on a panel of breast cancer cell lines. Using tissue microarrays and IHC, RBM3 protein expression was examined in $\mathbf{4 8}$ normal tissues and in $\mathbf{2 0}$ common cancers. Additional analysis in two independent breast cancer cohorts $(n=1016)$ with long-term follow-up was also carried out. RBM3 was upregulated in cancer compared to normal tissues. The nuclear expression of RBM3 in breast cancer was associated with low grade $(P<0.001)$, small tumors $(P<0.001)$, estrogen receptor $(E R)$ positivity $(P<0.001)$ and Ki-67 negativity $(P<0.001)$ in both the breast cancer cohorts. An increased nuclear expression of RBM3 was associated with a prolonged overall and recurrence-free survival. The prognostic value was particularly pronounced in hormone receptor-positive tumors and remained significant in multivariate interaction analysis after controlling for tamoxifen treatment (HR: $0.49,95 \% \mathrm{Cl}$ : $0.30-0.79, P=0.004)$. These data strongly indicate that nuclear RBM3 is an independent favorable prognostic factor in breast cancer, and seems to have a specific role in ER-positive tumors.

Modern Pathology (2009) 22, 1564-1574; doi:10.1038/modpathol.2009.124; published online 4 September 2009
\end{abstract}

Keywords: RBM3; breast cancer; prognosis; estrogen receptor; antibody-based proteomics

Single-strand RNA-binding proteins (RBPs) are involved in many aspects of RNA metabolism and

Correspondence: Professor K Jirström, Department of Laboratory Medicine, Center for Molecular Pathology, Malmö University Hospital, Lund University, SE-205 02 Malmö, Sweden.

E-mail: karin.jirstrom@med.lu.se

Received 7 April 2009; revised 21 July 2009; accepted 22 July 2009; published online 4 September 2009 in the regulation of gene transcription. ${ }^{1,2}$ Reversible binding of RBPs to RNA is accomplished through specific sequences within the proteins, such as the 'RNA-recognition motif' (RRM), 'RNA-binding motif' (RBM), 'ribonucleoprotein motif' (RNP), the arginine-rich motif (ARM), the cold-shock domain (CSD) and many others. ${ }^{2}$ RBM proteins have been recognized as a specific subgroup of RRM/ RBM/RNP containing RBPs. Each of the 10 
proteins designated as RBM proteins contain between 1 and 4 copies of the RRM consensus sequence. $^{1}$ The functional importance of the RRM domain is suggested by its evolutionary conservation across species and by its presence in virtually every organelle of the cell in which RNA is present. $^{1}$

Initially identified in a human fetal brain tissue cDNA library, ${ }^{3}$ the $R B M 3$ gene maps to Xp11.23, and is one of three X-chromosome-related RBM genes ( $R B M X, R B M 3$ and $R B M 10$ ). The $R B M 3$ gene encodes alternatively spliced transcripts, with the longest reading frame encoding for a 157 amino-acid protein containing one RRM domain and a glycine-rich region. ${ }^{3}$ The RBM3 protein binds to both DNA and RNA. ${ }^{4}$

Although RBM proteins have been proposed to be a novel family of apoptosis regulators, ${ }^{1,5-7}$ the exact function of RBM3 has yet to be fully elucidated. $R B M 3$ transcripts have been found in various human tissues, ${ }^{3}$ and in vitro, RBM3 is one of the earliest proteins synthesized in response to mild hypothermia. ${ }^{8}$ A correlation between the X chromosomerelated $R B M$ genes ( $R B M X, R B M 3$ and $R B M 10$ ) and the proapoptotic $B a x$ gene has been shown in breast cancer. ${ }^{5}$ Downregulation of $R B M 3$ is associated with melanoma progression; ${ }^{9}$ however, a recent report described $R B M 3$ as a proto-oncogene that protects against mitotic catastrophe in colorectal cancer cell lines. ${ }^{10}$ Taken together, the role of $R B M 3$ in the context of tumor initiation and progression in various cancer forms remains unknown and, to date, no studies have addressed the role of tumor-specific RBM3 protein expression in relation to disease outcome.

Using an antibody-based proteomics strategy, a comprehensive atlas of human protein expression patterns has been generated through the Human Protein Atlas program (http://www.proteinatlas. org). ${ }^{11,12}$ To date, more than 3000 antibodies (corresponding to more than 2600 different human proteins) have been screened in tissue microarrays, representing 48 types of normal tissues, 216 human tumor samples representing the 20 most common forms of human cancer and 47 cell lines, ${ }^{13,14}$ In addition to generating a map of protein expression patterns, this atlas can also be used as a platform for the discovery of new diagnostic, prognostic and predictive cancer biomarkers. ${ }^{12}$ One such candidate is RBM3, which, on the basis of its differential expression among the breast cancer samples in the protein atlas was further evaluated in tissue microarrays constructed from two independent breast cancer cohorts-one consecutive cohort $(n=512)$ and one cohort containing tumors from a randomized tamoxifen trial conducted in premenopausal women $(n=500)$. The latter cohort enabled assessment of the prognostic impact of RBM3 expression and also its potential value as a predictor of response to tamoxifen treatment.

\section{Materials and methods}

\section{Breast Cancer Cohorts}

Consecutive cohort (cohort I)

A total of 512 consecutive cases of primary breast cancer were diagnosed between 1988 and 1992 at the Department of Pathology, Malmö University Hospital. The median age at diagnosis was 64.2 years (range: 27-96), and the median follow-up time from diagnosis until the first breast cancer event was 106 months (range: 0-207). This cohort is described in detail elsewhere. ${ }^{15,16}$ Ethical permission was obtained from the local ethics committee at Lund University (Dnr 613/02).

Premenopausal randomized tamoxifen trial (cohort II) A total of 564 premenopausal women with stage II (pT2N0, pT1N1M0 and pT2N1M0) invasive breast cancer were enrolled in a randomized trial of 2 years tamoxifen treatment vs control between 1984 and 1991. ${ }^{17}$ The median follow-up for patients without a breast cancer event was 13.9 years. The study design is described in detail elsewhere. ${ }^{18,19}$ Tissue microarrays were constructed from 500 available tumor specimens. Ethical permission was obtained from the ethics committees at Lund and Linköping Universities.

\section{Tissue Microarray Construction}

HPA-screening tissue microarrays were constructed as described previously. ${ }^{13}$ Before tissue microarray construction from the extended cohorts, all breast cancer cases were histopathologically re-evaluated on hematoxylin \& eosin-stained slides. Areas representative of cancer were then marked and tissue microarrays were constructed as described previously $^{20}$ using either a manual tissue arrayer (MTA-1, Beecher, WI, USA) (cohort I) or an automated tissue arrayer (ATA-27, Beecher) (cohort II). Two $1.0-\mathrm{mm}$ cores (cohort I) or $0.6-\mathrm{mm}$ cores (cohort II) were extracted from each donor block and assembled in a recipient block as described previously. ${ }^{19}$

\section{RBM3 Antibody Generation and Immunohistochemistry}

A mono-specific Protein Epitope Signature Tags antibody was generated using a recombinant protein fragment corresponding to the human RBM3 gene (HPA 3624) as described previously. ${ }^{21}$ Sections of $4-\mu \mathrm{m}$ thickness were dried, deparaffinized and rehydrated. After heat-mediated antigen retrieval at pH 9.9 (Target Retrieval Solution, Dako, Glostrup, Denmark), IHC was performed in Techmate 500 (Dako) using the anti-RBM3 (HPA 3624) antibody at a dilution of 1:100. For all other antibodies, heatmediated antigen retrieval was performed using 
microwave treatment for $2 \times 5 \mathrm{~min}$ in a citrate buffer before being processed either in the Ventana Benchmark system (Ventana Medical Systems, AZ, USA) using pre-diluted antibodies to estrogen receptor (ER) (Anti-ER, clone 6F11), progesterone receptor (PR) (Anti-PgR, clone 16) and Her2 (Pathway CBUSA, 760-2694) or in the Techmate 500 system (Dako) for Ki-67 (1:200, M7240; Dako). DAB (3,3'diaminobenzidine) was used as a chromogenic substrate and slides were counterstained using hematoxylin.

Nuclear and cytoplasmic RBM3 expressions were assessed using the same scoring system as that used in the web-based annotation of the HPA. ${ }^{22}$ Nuclear and cytoplasmic staining fractions were assigned a score from $0(0-1 \%), 1(2-25 \%), 2(26-75)$ to 3 $(>75 \%)$ and the staining intensities within the respective subcellular locations were noted as $0=$ negative, $1=$ weak, $2=$ moderate and $3=$ strong. ER, PR, HER2 and Ki-67 were assessed as described previously. ${ }^{15}$ ER and PR negativity was defined as $<10 \%$ positively staining nuclei, according to the current clinical guidelines in Sweden.

\section{Cell Culture and Gene Silencing}

The human breast cancer cell lines, MCF-7, MDAMB-231, T47D, and the human immortalized breast epithelial cell line MCF-10A were maintained under standard conditions. Transfection with siRNA against RBM3 (Applied Biosystems, Carlsbad, CA, USA) or control siRNA (Applied Biosystems), was performed with Lipofectamine 2000 (Invitrogen, Carlsbad, CA, USA) with a final concentration of $50 \mathrm{nM}$ siRNA. Cells were harvested for protein or RNA recovery 48 and $72 \mathrm{~h}$ post transfection initiation. IHC was performed on cells after fixation in paraformaldehyde $(4 \%)$, dehydration and paraffin embedding.

\section{PCR and Western Blotting}

RNA isolation (RNeasy, Qiagen), complementary DNA generation (Reverse Transcriptase Kit, Applied Biosystems) and quantitative real-time PCR (qPCR) (SYBR green PCR mastermix, Applied Biosystems) were performed as described previously. ${ }^{23,24}$ For immunoblotting, cells were lysed in RIPA buffer (10 mM Tris-HCl pH 7.2, $160 \mathrm{mM} \mathrm{NaCl,} 1 \%$ Triton-X, $1 \%$ sodium deoxycholate, $0.1 \%$ SDS, $1 \mathrm{nM}$ EDTA, $1 \mathrm{mM}$ EGTA) and supplemented with protease inhibitor cocktail (Roche Applied Science, Burgess Hill, West Sussex, UK). For each sample $60 \mu \mathrm{g}$ of protein was separated by $10 \%$ SDS-PAGE and blotted onto PVDF membranes (Millipore, Billerica, MA, USA). RBM3 was detected by HPA 3624 diluted $1: 250$ in $5 \%$ non-fat milk PBST.

\section{Statistics}

Chi-square test was used for comparison of RBM3 expression and relevant clinico-patholgical characteristics. Kaplan-Meier analysis and log-rank test were used to illustrate differences in both recurrencefree and overall survival according to the RBM3 expression. Recurrence-free survival considered locoregional and distant metastasis or breast cancerspecific death as the primary event, whereas contralateral events were excluded. Cox regression proportional hazards models were used to estimate the impact of RBM3 expression on recurrence-free and overall survival in both univariate and multivariate analysis, after adjusting for tumor size, age at diagnosis, ER, HER2, lymph-node status and Nottingham Histological Grade. All calculations were performed using SPSS version 15.0 (SPSS, Chicago, IL, USA). All statistical tests were two-sided and a $P$-value $<0.05$ was considered statistically significant.

\section{Results}

\section{RBM3 Protein Expression in Normal and Tumor Tissues}

Antibody-based screening of RBM3 in 48 different human tissues showed moderate nuclear and/or cytoplasmic expression in the breast and epididymal epithelium, lung macrophages, skeletal muscle, parathyroid, placenta, skin, testes and tonsil (Figure 1). In a normal breast, scattered expression of RBM3 was observed in luminal rather than in myoepithelial cells (Figure 1c). Analysis in 20 different cancer types showed increased expression of RBM3 in breast cancer (10 of 12) (Figure 1). Increased RBM3 expression was also evident in malignant carcinoid ( 3 of 4), testicular cancer (6 of 12) and in non-melanoma skin cancer (9 of 11) (Figure 1).

\section{Expression of RBM3 in Human Breast Cancer and Breast Cancer Cell Lines}

RBM3 expression was examined in a panel of cell lines-MCF-7, T47D, MDA-MB-231 and MCF10A. Western blot analysis using the anti-RBM3 antibody recognized a single band at $17 \mathrm{kDa}$ (Figure 2a). The differential expression of RBM3 was evident, with highest levels observed in MCF-7 cells (Figure 2a). IHC performed on the cell lines confirmed the western blot findings (Figure 2a). Western blot analysis of protein extracts collected from six invasive ER-positive breast tumors showed that RBM3 was expressed in four samples (Figure 2b).

Antibody specificity was confirmed using siRNA-mediated gene silencing of RBM3 performed on MCF-7 and MDA-MB-231 cell lines. RBM3 
a

\begin{tabular}{|c|c|c|c|}
\hline $\begin{array}{l}\text { Adrenal aland } \\
\text { ADoendix }\end{array}$ & $\begin{array}{l}\text { cortical cels } \\
\text { vandular cels } \\
\text { vempheid tissue }\end{array}$ & $\begin{array}{l}\text { Lymoh node } \\
\text { Nasopharynx }\end{array}$ & 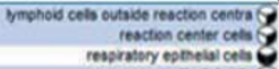 \\
\hline Dene marrow & boee marrow pobetic celt 8 & eral mucesa & 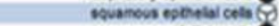 \\
\hline $\begin{array}{l}\text { Breast } \\
\text { Bronchus }\end{array}$ & 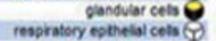 & Ovarx & 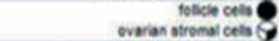 \\
\hline Serebellum & 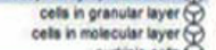 & Pancreas & 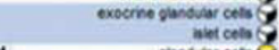 \\
\hline Serebral certex & 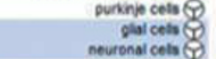 & $\begin{array}{l}\text { Parathuroid aland } \\
\text { Placenta }\end{array}$ & 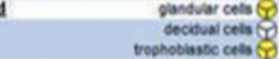 \\
\hline Cenxix, uterine & 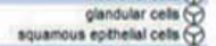 & $\begin{array}{l}\text { Prostate } \\
\text { Bectum }\end{array}$ & ginoviar celis of \\
\hline Colon & siandviar celto 0 & Salixary aland & dianoviar cele 85 \\
\hline Corous, uterine 1 & 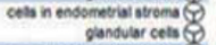 & $\begin{array}{l}\text { Seminal resicle } \\
\text { Skeletal muscle }\end{array}$ & 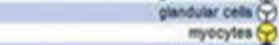 \\
\hline Corpus, vterine 2 & 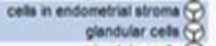 & Skin & 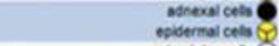 \\
\hline Duodenum & vlandular celts 6 & Sma & glanoviar celis 6 \\
\hline Epidis & giandvar cols 6 & Smo & mectin nuacie celly \\
\hline Esophaqus & aquamous epthelal celts & Softet & mesenchymai cells of \\
\hline Eallepian tube & dianoviar cels of & Softetiss: & mesenctiy \\
\hline $\begin{array}{l}\text { Gall blads } \\
\text { Heartmu }\end{array}$ & sandvar cels of & Spleen & celis in ros pub of \\
\hline $\begin{array}{l}\text { Heart muscle } \\
\text { Hippocampus }\end{array}$ & poctur cels of & Stem & $\begin{array}{l}\text { colls in whte pub of } \\
\text { plandviar cats of }\end{array}$ \\
\hline & neuronal c & Ste: & glandviar cells \&? \\
\hline Kidnex & 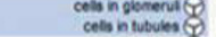 & Iestis & 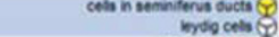 \\
\hline Lateral ventricle & vial cets 8 & $\begin{array}{l}\text { Throoid aland } \\
\text { Tonsil }\end{array}$ & 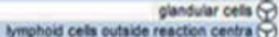 \\
\hline Liver & Whe duct cello of & & 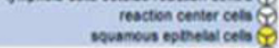 \\
\hline Lung & $\begin{array}{l}\text { aveour cets } 89 \\
\text { macrophapen } 8\end{array}$ & $\begin{array}{l}\text { Yrinary bladder } \\
\text { Yaaina } \\
\text { Yulva/anal skin }\end{array}$ & 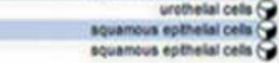 \\
\hline
\end{tabular}

b

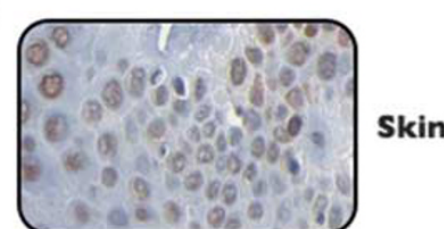

C

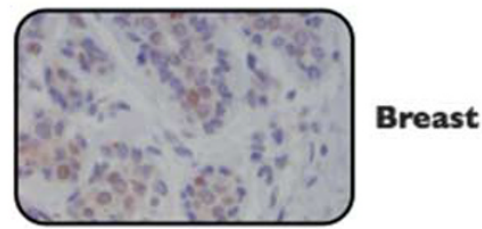

d

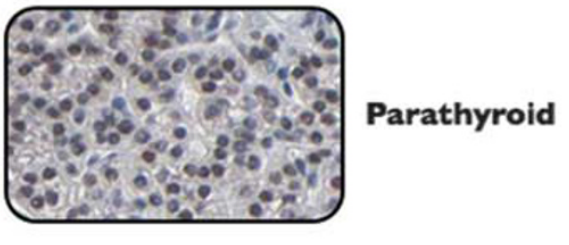

e

\begin{tabular}{|c|c|}
\hline \multirow{2}{*}{\multicolumn{2}{|c|}{$\begin{array}{l}\text { Colorectal cancer } \\
\text { Breast cancer }\end{array}$}} \\
\hline & \\
\hline \multicolumn{2}{|l|}{ Prostate cancer } \\
\hline \multirow{2}{*}{\multicolumn{2}{|c|}{$\begin{array}{l}\text { Ovarian cancer } \\
\text { Cervical cancer }\end{array}$}} \\
\hline & \\
\hline \multicolumn{2}{|l|}{ Endemetrial cancer } \\
\hline \multicolumn{2}{|l|}{ Malianant carcinoid } \\
\hline \multicolumn{2}{|l|}{ Head \& neck cancer } \\
\hline \multicolumn{2}{|l|}{ Thyroid cancer } \\
\hline \multicolumn{2}{|l|}{ Malionant glioma } \\
\hline \multicolumn{2}{|c|}{ Maliqnant Iymphoma } \\
\hline \multicolumn{2}{|l|}{ Lung cancer } \\
\hline \multicolumn{2}{|c|}{ Malianant melanoma } \\
\hline \multicolumn{2}{|l|}{ Skin cancer } \\
\hline \multicolumn{2}{|l|}{ Iestis cancer } \\
\hline \multicolumn{2}{|l|}{ Urothelial cancer } \\
\hline \multicolumn{2}{|l|}{ Renal cancer } \\
\hline \multicolumn{2}{|l|}{$\begin{array}{l}\text { Stomach cancer } \\
\text { Pancreatic cancer }\end{array}$} \\
\hline Liver cancer & \\
\hline
\end{tabular}

$f$

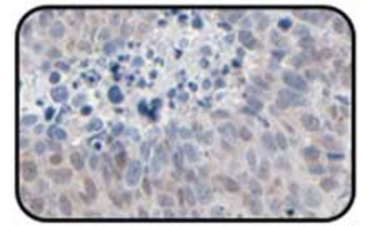

g

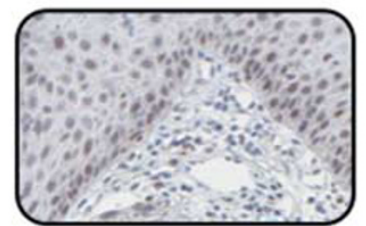

h

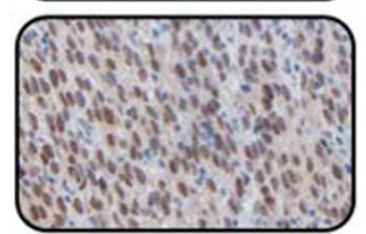

Malignant Carcinoid

\section{Skin Cancer}

\section{Testis Cancer}

\section{RBM3}

strong positivity
moderate positivity
no values attributable to the given specimen

Figure 1 Expression pattern of RBM3 protein in normal and cancer tissues. A summary view showing levels of RBM3 protein expression in a panel of normal human tissues (a). Mild-to-moderate positivity (yellow) was observed in the skin (b), breast epithelium (c) and parathyroid (d). Lung macrophages, skeletal muscle, placenta, testes and tonsil also showed low levels of RBM3 protein expression. A summary view showing expression of RBM3 protein in 216 cancers from 20 different tumor types (e). The high frequency of moderate expression (yellow) in breast cancer must be noted. RBM3 expression was also evident in malignant carcinoid (f), non-melanoma skin cancer (g) and testicular cancer (h). Other tumor types showed lower levels of RBM3 protein expression.

gene expression was successfully reduced after transfection with two different siRNA sequences (siRNA 58 and 59) as shown by qPCR at $72 \mathrm{~h}$ post transfection (Supplementary Figure 1). Gene silencing was also confirmed by western blot analysis (Figure 2c). Significantly, IHC staining for RBM3 of cells transfected with siRNA against $R B M 3$ and control siRNA showed specific loss of signal in the cells transfected with siRNA against RBM3 compared with control RNA or no siRNA (Figure 2d). The loss of RBM3 signal with siRNAmediated knockdown corroborates the specificity of the antibody in both IHC and immunoblot applications.

\section{Nuclear RBM3 Expression in Breast Cancer is Associated with Favorable Clinico-Pathological Parameters}

RBM3 protein expression was then examined in two independent extended breast cancer cohorts. RBM3 was present in various fractions and intensities in tumor cell nuclei, whereas cytoplasmic staining, when present, was evident in the majority of tumor cells in varying intensities (Figure 2e). After antibody optimization and immunohistochemical processing, RBM3 protein expression was evaluable in $241(47 \%)$ cases in cohort I and in $311(61 \%)$ cases in cohort II. To evaluate our study for any potential 

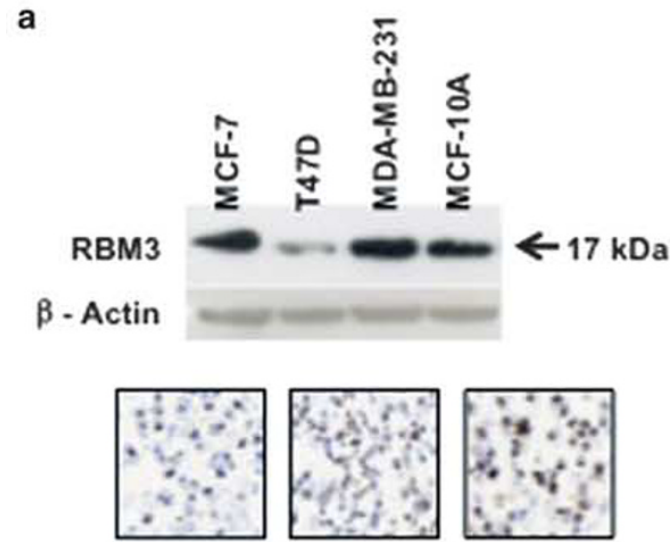

MCF-7

T47D

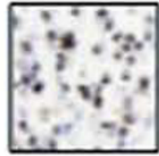

MDA-MB

231

b

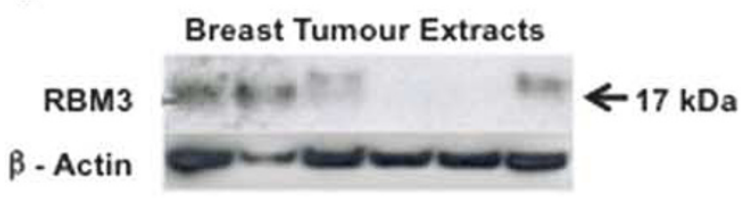

C
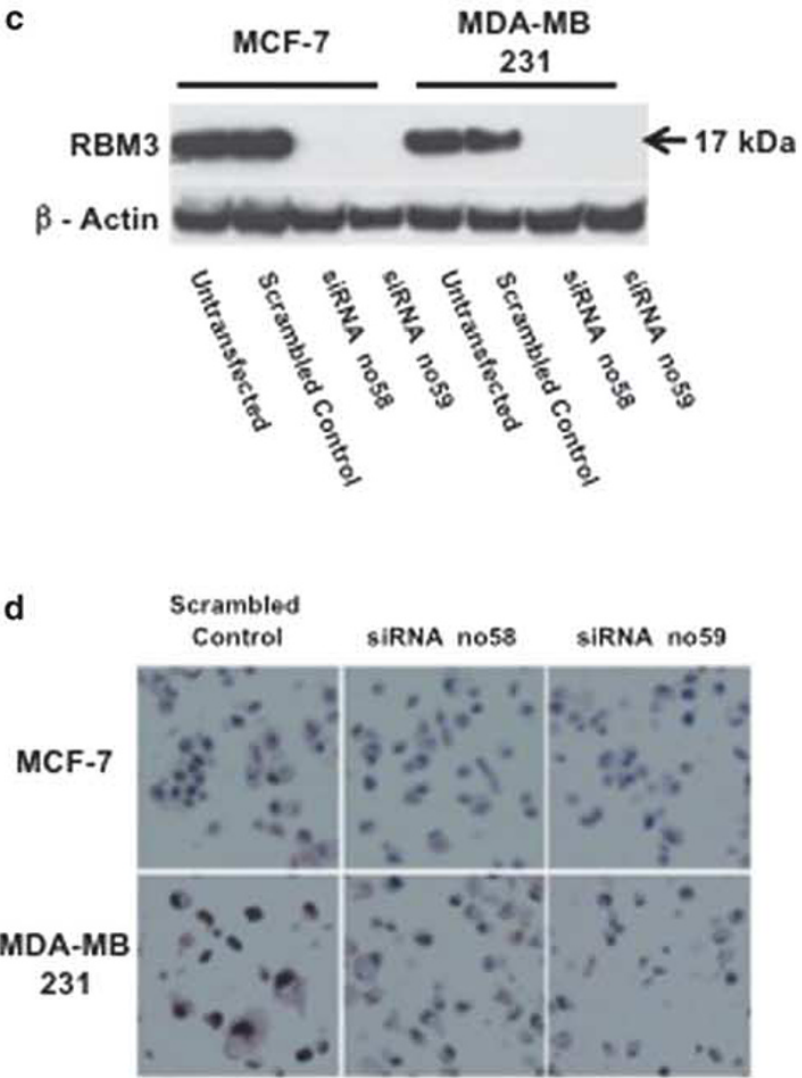

e
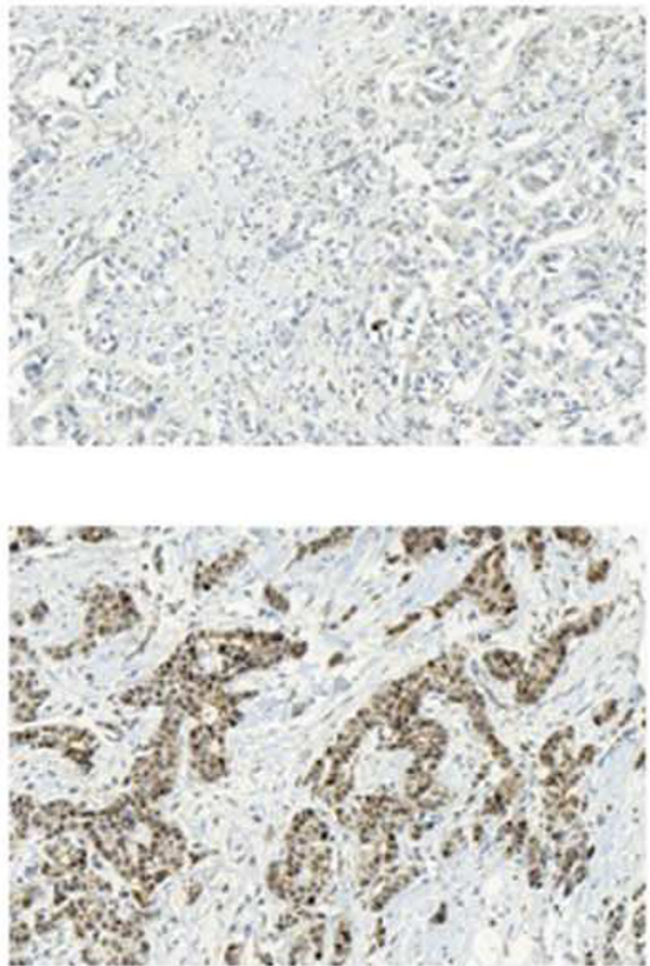
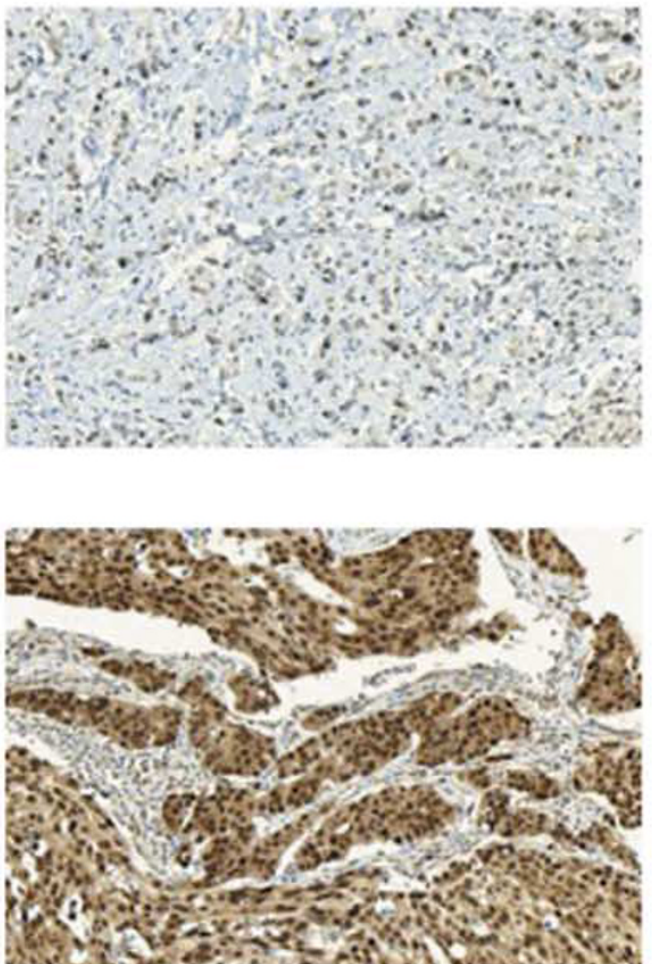
selection bias, baseline clinicopathological characteristics from the evaluated or 'RBM3-known' cases and from the unevaluated or 'RBM3-unknown' cases in both are presented in Supplementary Table 1. This shows that there were significantly more ERand PR-positive tumors in the 'RBM3-known' cases in cohort I, otherwise no significant differences were evident between the 'RBM3-known' and 'RBM3unknown' tumors in both cohorts, indicating the absence of any selection bias.

In cohort I, 150 tumors (62\%) expressed high levels of RBM3 ( $>75 \%$ positive nuclei), whereas in cohort II only 121 tumors (39\%) expressed high levels of RBM3. This could be attributed to the fact that cohort II consisted of exclusively premenopausal patients, and also contained a larger proportion of high-grade, ER-negative tumors. Despite these differences, the nuclear expression of RBM3 was associated with a number of favorable clinicopathological parameters in both breast cancer cohorts, such as small tumor size, low grade, Ki-67 negativity as well as ER and PR positivity (Table 1). No association was evident between nuclear RBM3 expression and Her2 or nodal status. In both cohorts, a significant correlation between nuclear and cytoplasmic staining was evident $(R=0.154, P=0.016$ for cohort I and $R=0.214, P<0.001$ for cohort II), but apart from a positive correlation with ER positivity in both cohorts $(P=0.04$ and $P=0.02$, respectively) and $\mathrm{PR}$ positivity in cohort II $(P=0.01)$, cytoplasmic staining was not associated with any relevant clinicopathological parameters.

\section{RBM3 Expression is an Independent Predictor of Disease Outcome in ER-Positive Breast Cancer}

Having shown that RBM3 nuclear expression was associated with a less aggressive phenotype, the relationship between RBM3 protein expression and disease outcome in two independent breast cancer cohorts was examined. Nuclear, but not cytoplasmic, expression was associated with improved survival and analysis of different thresholds showed an optimal cutoff at $75 \%$ positivity in both cohorts (Figure 3). RBM3 protein expression data were thus dichotomized into $<75 \%$ vs $>75 \%$ positive nuclear staining and this dichotomized variable was used for further statistical analyses. Kaplan-Meier analysis showed that increased levels of RBM3, using a cutoff of $75 \%$ positive nuclei, was associated with an improved OS in cohort I $(P=0.001)$ and in cohort II $(P=0.006)$ (Figure 3).

Cox regression univariate analysis showed that increased RBM3 nuclear expression $(>75 \%)$ was associated with an improved recurrence-free survival $(\mathrm{RR}=0.58,95 \% \mathrm{CI}$ : $0.36-0.95, P=0.03)$ and OS $(\mathrm{RR}=0.55,95 \%$ CI: $0.38-0.78, P=0.001)$ in cohort I (Table 2). Multivariate analysis within the same cohort showed that increased expression of nuclear RBM3 was an independent predictor of an improved recurrence-free survival $(\mathrm{RR}=0.52,95 \% \mathrm{CI}: 0.27-$ $0.99, P=0.05)$ and overall survival $(\mathrm{RR}=0.56,95 \%$ CI: $0.34-0.92, P=0.02$ ) after controlling for grade, nodal status, tumor size, ER, PR and Her2 status (Table 2). Given the previous observation of a possible relationship between RBM3 expression and ER positivity (Table 1), the relationship between RBM3 and outcome in ER-positive tumors was examined and RBM3 was identified as an independent predictor of improved overall survival in ERpositive patients $(\mathrm{RR}=0.49,95 \% \mathrm{CI}$ : $0.29-0.83$, $P=0.008$ ) (Table 2). RBM3 had no impact on outcome in hormone receptor-negative patients (data not shown).

Increased RBM3 nuclear expression ( $>75 \%$ ) was also associated with an improved recurrence-free survival $(\mathrm{RR}=0.70,95 \% \mathrm{CI}$ : $0.50-0.99, P=0.04)$ and OS $(\mathrm{RR}=0.61,95 \%$ CI: $0.42-0.87, P=0.006)$ in cohort II. Cox multivariate analysis showed that RBM3 was not an independent predictor of recurrence-free or overall survival in cohort II (Table 3). However, a subgroup analysis of ER-positive patients using Cox multivariate regression analysis adjusted for treatment bias revealed that increased RBM3 nuclear expression ( $>75 \%$ ) was associated with an improvement in recurrence-free survival $(\mathrm{RR}=0.56,95 \%$ CI: $0.36-0.90, P=0.02)$ and OS $(\mathrm{RR}=0.49,95 \%$ CI: $0.30-0.79, P=0.004)$ (Table 3 ). Given these findings, the relationship between RBM3 expression and tamoxifen response in cohort II was investigated. As recurrence-free survival was the end point of the original trial, this was used in this analysis. Kaplan-Meier analysis that compared 2 years adjuvant tamoxifen to no systemic treatment (control), stratified according to RBM3 expression, showed no relationship between RBM3 expression and tamoxifen response in premenopausal breast cancer (Figures 3e and f).

Figure 2 RBM3 is expressed in human breast-derived cell lines and breast tumors. Western blotting in a panel of human cell lines, breast cancer derived MCF-7, T47D and MDA-MB-231, and the immortalized breast epithelial cell line MCF-10A recognized the 17-kDa RBM3 protein (a, upper panel). Western blot was confirmed using IHC on MCF-7, MDA-MB-231 and T47D cell lines. RBM3 expression was confined to the nuclei in MCF-7 and MDA-MB-231 cells, whereas cytoplasmic expression was also evident in T47D cells (panel a, lower panel). Western blot of protein extracts from six breast tumors detected with anti-RBM3 (lower panel). In all, $60 \mu \mathrm{g}$ protein was separated by SDS-PAGE and blotted onto PVDF membranes. Below each panel, actin staining of the same membranes is shown (b). Antibody specificity was confirmed using siRNA-mediated gene silencing. Two different commercially available (Applied Biochemistry) siRNAs against RBM3 and control siRNA was transfected into MCF-7 and MDA-MB-231 cells, and the RBM3 protein expression was assessed at $72 \mathrm{~h}$ using western blotting (c). IHC staining of siRNA-transfected cells (d). MCF-7 and MDA-MB-231 cells transfected with control siRNA or siRNA against RBM3 (siRNA58 and siRNA59). IHC was performed analogous to that on tissue microarrays. Examples of tumors with negative, low, moderate and high levels of RBM3 expression (e). 
Table 1 Relationship between RBM3 and clinicopathological parameters in two breast cancer cohorts

\begin{tabular}{|c|c|c|c|c|c|c|c|c|c|c|}
\hline \multirow{2}{*}{$\begin{array}{l}\text { RBM3 nuclear fraction } \\
\mathrm{n}(\%)\end{array}$} & \multicolumn{5}{|c|}{ Cohort I n $=512$ (241) } & \multicolumn{5}{|c|}{ Cohort II $\mathrm{n}=500$} \\
\hline & $\begin{array}{c}0-1 \% \\
19(7.9)\end{array}$ & $\begin{array}{l}2-25 \% \\
26(10.8)\end{array}$ & $\begin{array}{l}26-75 \% \\
46(19.1)\end{array}$ & $\begin{array}{c}>75 \% \\
150(62.2)\end{array}$ & $\mathrm{P}$-value & $\begin{array}{c}0-1 \% \\
54(17.4)\end{array}$ & $\begin{array}{c}2-25 \% \\
58(18.6)\end{array}$ & $\begin{array}{l}26-75 \% \\
78(25.1)\end{array}$ & $\begin{array}{c}>75 \% \\
121(38.9)\end{array}$ & $\mathrm{P}$-value \\
\hline \multicolumn{11}{|l|}{ Tumor size } \\
\hline$\leq 20 \mathrm{~mm}$ & $7(36.8)$ & 14 (53.9) & $26(56.5)$ & $106(70.7)$ & 0.001 & $7(13.0)$ & $18(31.0)$ & 31 (39.7) & $51(42.2)$ & 0.001 \\
\hline$>20 \mathrm{~mm}$ & $12(63.2)$ & $12(46.1)$ & $20(43.5)$ & $44(29.3)$ & & $47(87.0)$ & $40(69.0)$ & $47(60.3)$ & $69(57.0)$ & \\
\hline Missing & $0(0)$ & $0(0)$ & $0(0)$ & $0(0)$ & & $0(0)$ & $0(0)$ & $0(0)$ & $1(0.8)$ & \\
\hline \multicolumn{11}{|l|}{ Grade } \\
\hline I & $1(5.3)$ & $4(15.4)$ & $7(15.2)$ & $40(26.6)$ & $<0.001$ & $2(3.7)$ & $2(3.5)$ & $10(12.8)$ & $16(13.2)$ & $<0.001$ \\
\hline II & $3(15.8)$ & 14 (53.8) & 19 (41.3) & $73(48.7)$ & & 10 (18.5) & $14(24.1)$ & $37(47.4)$ & $66(54.6)$ & \\
\hline III & 15 (78.9) & $17(65.4)$ & $20(43.5)$ & $37(24.7)$ & & $41(75.9)$ & $39(67.2)$ & 31 (39.7) & 37 (30.6) & \\
\hline Missing & $0(0)$ & $0(0)$ & $0(0)$ & $0(0)$ & & $1(1.9)$ & $3(5.2)$ & $0(0)$ & $2(1.6)$ & \\
\hline \multicolumn{11}{|l|}{ Node status } \\
\hline Negative & $10(52.6)$ & $14(53.8)$ & $23(50.0)$ & $91(60.7)$ & 0.30 & $19(35.2)$ & $19(32.8)$ & $18(23.1)$ & $29(24.0)$ & 0.10 \\
\hline Positive & $7(36.8)$ & $9(34.6)$ & 19 (41.3) & $45(30.0)$ & & $35(64.8)$ & $39(67.2)$ & 60 (76.9) & $91(75.2)$ & \\
\hline Missing & $2(10.5)$ & $3(11.5)$ & $4(8.7)$ & $14(9.3)$ & & $0(0)$ & $0(0)$ & $0(0)$ & $1(0.8)$ & \\
\hline \multicolumn{11}{|l|}{ ER status } \\
\hline Negative & $9(47.4)$ & $3(11.5)$ & $5(10.9)$ & $8(5.3)$ & $<0.001$ & $40(74.1)$ & $28(48.3)$ & 17 (21.8) & 19 (15.7) & $<0.001$ \\
\hline Positive & $10(52.6)$ & 23 (88.5) & 41 (89.1) & $138(92.0)$ & & $13(24.1)$ & $28(48.3)$ & $58(74.4)$ & $97(80.2)$ & \\
\hline Missing & $0(0)$ & $0(0)$ & $0(0)$ & $4(2.7)$ & & $1(1.8)$ & $2(3.4)$ & $3(3.8)$ & $5(4.1)$ & \\
\hline \multicolumn{11}{|l|}{ PR status } \\
\hline Negative & $12(63.2)$ & $6(23.1)$ & $8(17.4)$ & $34(22.7)$ & 0.015 & $38(70.4)$ & $29(50.0)$ & $22(28.2)$ & $23(19.0)$ & $<0.001$ \\
\hline Positive & $5(26.3)$ & 14 (53.8) & $30(65.2)$ & $81(54.0)$ & & $13(24.1)$ & $26(44.8)$ & $52(66.7)$ & 89 (73.6) & \\
\hline Missing & $2(10.5)$ & $6(23.1)$ & $8(17.4)$ & $35(23.3)$ & & $3(5.5)$ & $3(5.2)$ & $4(5.1)$ & $9(7.4)$ & \\
\hline \multicolumn{11}{|l|}{ HER2 (IHC) } \\
\hline 0 & $13(6.8)$ & 15 (57.8) & 19 (41.3) & 76 (51.0) & 0.88 & $36(66.7)$ & $31(53.4)$ & $43(55.1)$ & $60(49.6)$ & 0.38 \\
\hline $1+$ & $1(5.3)$ & $4(15.4)$ & 13 (28.3) & $40(26.7)$ & & $6(11.1)$ & 7 (12.1) & 14 (17.9) & $17(14.0)$ & \\
\hline $2+$ & $3(15.8)$ & $4(15.4)$ & $6(13.0)$ & $10(6.7)$ & & $1(1.8)$ & $4(6.9)$ & $7(9.0)$ & $9(7.4)$ & \\
\hline $3+$ & $2(10.5)$ & $1(3.8)$ & 510.9) & $11(7.3)$ & & $8(14.8)$ & $9(15.5)$ & $8(10.3)$ & $17(14.1)$ & \\
\hline Missing & $0(0)$ & $2(7.7)$ & $3(6.5)$ & $13(8.7)$ & & $3(7.6)$ & $7(12.1)$ & $6(7.7)$ & 18 (14.9) & \\
\hline \multicolumn{11}{|l|}{ Ki67 (\%) } \\
\hline $0-10$ & $5(26.3)$ & $9(34.6)$ & $12(26.1)$ & $67(44.6)$ & $<0.001$ & $13(24.1)$ & $14(24.1)$ & $37(47.4)$ & $51(42.2)$ & $<0.001$ \\
\hline $11-25$ & $2(10.5)$ & $8(30.8)$ & 19 (41.3) & $52(34.7)$ & & 14 (25.9) & $18(31.0)$ & $20(25.6)$ & $34(28.1)$ & \\
\hline$>25$ & $12(53.2)$ & $9(34.6)$ & 15 (32.6) & $25(16.7)$ & & $23(42.6)$ & $22(37.9)$ & $18(23.1)$ & $16(13.2)$ & \\
\hline Missing & $0(0)$ & $0(0)$ & $0(0)$ & $6(4.0)$ & & $4(7.4)$ & $4(6.9)$ & $3(3.9)$ & $20(16.5)$ & \\
\hline
\end{tabular}

ER, estrogen receptor; PR, progesterone receptor; IHC, immunohistochemistry.

\section{Discussion}

This is the first study to evaluate tumor-specific expression of the RBP, RBM3. Using an antibodybased proteomics approach to explore RBM3 protein expression in normal tissues and tumors, we showed that RBM3 is seldom expressed in normal tissues, but is expressed in a number of common cancers (Figure 1). Immunohistochemistry performed on breast cancer tissue microarrays showed that nuclear RBM3 expression was associated with small, low-grade, ER-positive tumors in two independent breast cancer cohorts (Table 1). Increased nuclear expression of RBM3 protein was associated with an improved overall and recurrence-free survival in both cohorts, and a subset analysis of ER-positive patients showed that increased levels of RBM3 was an independent predictor of overall survival in cohort I (Table 2). In cohort II, RBM3 was an independent predictor of recurrence-free and overall survival in ER-positive patients after controlling for other established prognostic parameters and tamoxifen treatment (Table 3). Increased levels of nuclear RBM3 expression were not predictive of tamoxifen response in premenopausal breast cancer (Figure 3). These findings support the role of RBM3 as an important prognostic factor in breast cancer.

RBM3 is a cold-shock protein ${ }^{8}$ and the role of these proteins in tumor progression is still not fully understood. Cold shock has been suggested to be an important mediator of a novel type of mitotic cell death known as caspase-independent mitotic death $;^{25}$ however, the role of RBM3 in this process remains unknown. CSD protein $\mathrm{A}$ has also been shown to inhibit angiogenesis and lymphangiogenesis in a Lewis lung carcinoma xenograft mouse model. ${ }^{26}$ RBM3 may influence tumor progression by controlling global protein expression within the cell 
a

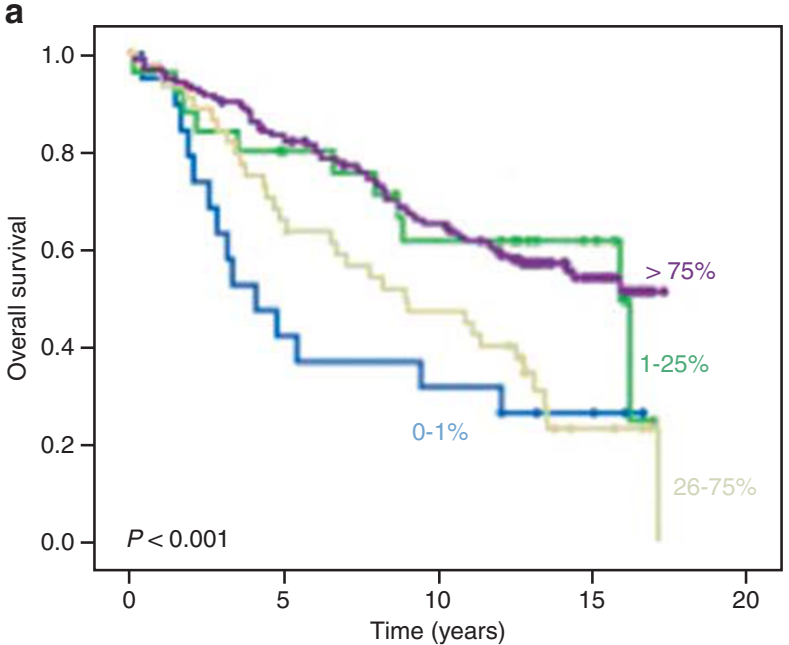

C

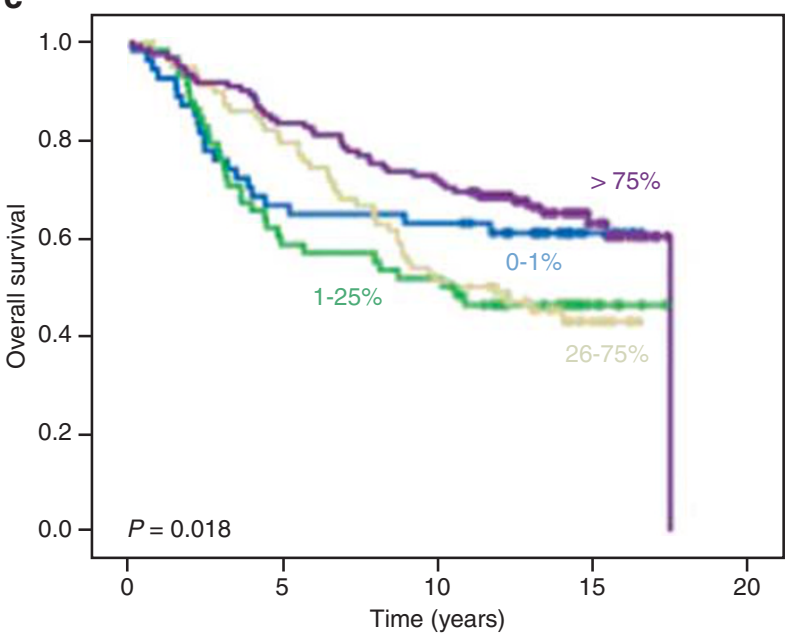

e

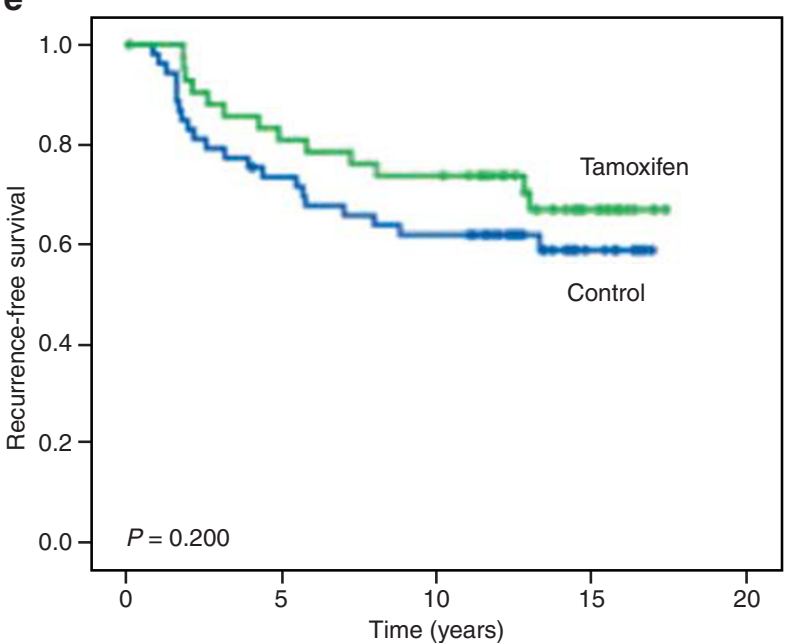

b

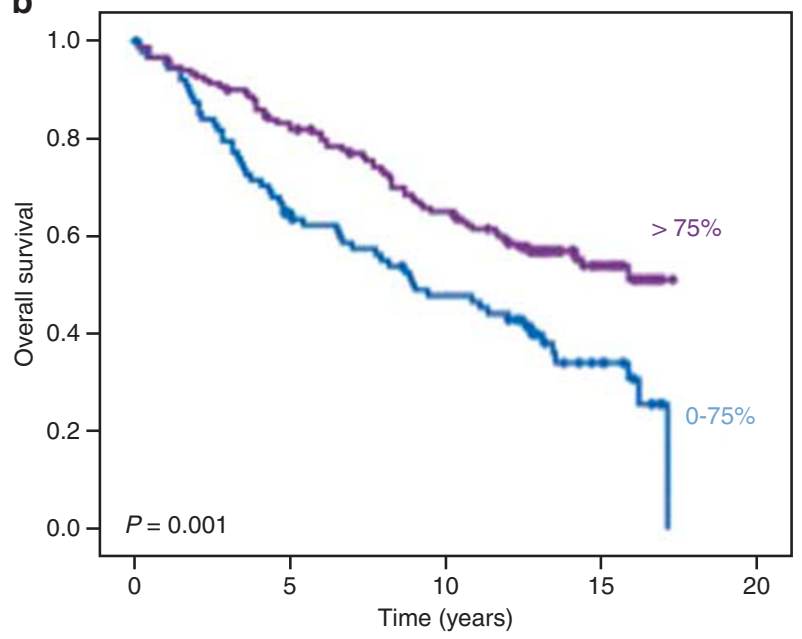

d

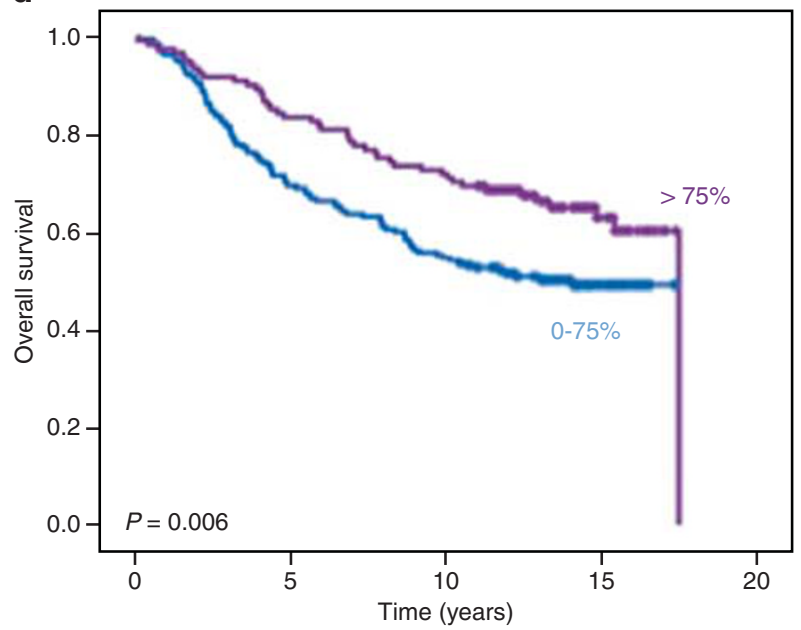

f

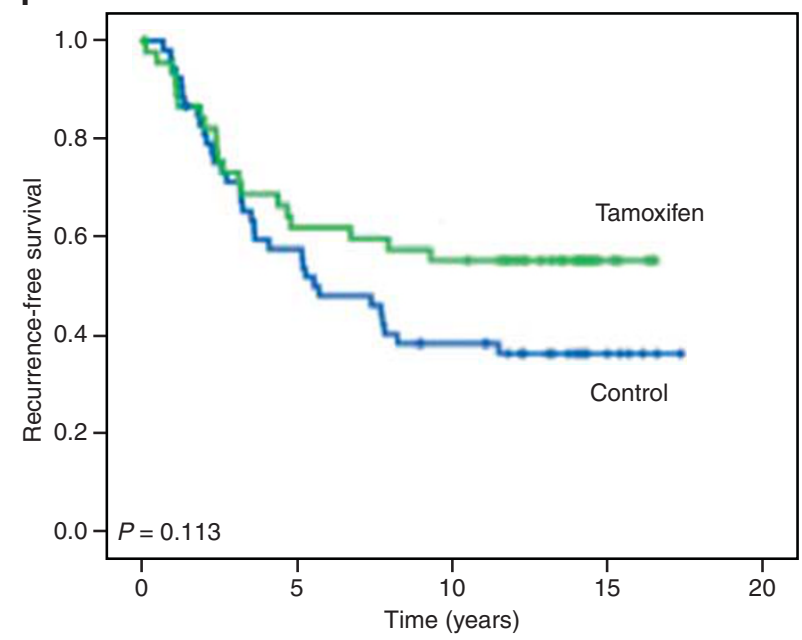

Figure 3 Nuclear expression of RBM3 is associated with an improved OS, but is not a predictor of tamoxifen response. Kaplan-Meier analysis of overall survival in cohort I (a and b) and in cohort II (c and d) showing 75\% positive nuclei as the optimal threshold for RBM3. Kaplan-Meier analysis of recurrence-free survival after 2 years tamoxifen compared with no adjuvant treatment (control) in premenopausal breast cancer identify no benefit for tamoxifen in tumors expressing high (e) or low (f) levels of RBM3. This analysis was confined to ER-positive patients in cohort II who had participated in a randomized control trial. 
Table 2 Cox univariate and multivariate analysis of recurrence-free and overall survival in cohort I

\begin{tabular}{|c|c|c|c|c|}
\hline & \multicolumn{2}{|c|}{ Recurrence-free survival } & \multicolumn{2}{|c|}{ Overall survival } \\
\hline & $R R(95 \% C I)$ & $\mathrm{P}$-value & $R R(95 \% C I)$ & $\mathrm{P}$-value \\
\hline \multicolumn{5}{|c|}{ All patients $(\mathrm{n}=241)$} \\
\hline RBM3 & \multicolumn{2}{|c|}{ Univariate } & \multicolumn{2}{|c|}{ Univariate } \\
\hline Low & 1.00 & & 1.00 & \\
\hline High & $0.58(0.36-0.95)$ & 0.03 & $0.55(0.38-0.78)$ & 0.001 \\
\hline RBM3 & \multicolumn{2}{|c|}{ Multivariate $^{\mathrm{a}}$} & \multicolumn{2}{|c|}{ Multivariate $^{\mathrm{a}}$} \\
\hline Low & 1.00 & & 1.00 & \\
\hline High & $0.52(0.27-0.99)$ & 0.05 & $0.56(0.34-0.92)$ & 0.02 \\
\hline \multicolumn{5}{|c|}{ ER-positive patients $(\mathrm{n}=210)$} \\
\hline RBM3 & \multicolumn{2}{|c|}{ Univariate } & \multicolumn{2}{|c|}{ Univariate } \\
\hline Low & 1.00 & & 1.00 & \\
\hline High & $0.63(0.37-1.08)$ & 0.095 & $0.54(0.37-0.80)$ & 0.002 \\
\hline RBM3 & \multicolumn{2}{|c|}{ Multivariate $^{\mathrm{a}}$} & \multicolumn{2}{|c|}{ Multivariate $^{\mathrm{a}}$} \\
\hline Low & 1.00 & & 1.00 & \\
\hline High & $0.54(0.27-1.11)$ & 0.092 & $0.49(0.29-0.83)$ & 0.008 \\
\hline
\end{tabular}

${ }^{\mathrm{a}}$ Multivariate analysis included adjustment for grade, age, nodal status, ER status, PR status, Her2 and tumor size.

Table 3 Cox regression analysis of recurrence-free and overall survival in different patient strata in cohort II

\begin{tabular}{|c|c|c|c|c|}
\hline & \multicolumn{2}{|c|}{ Recurrence-free survival } & \multicolumn{2}{|c|}{ Overall survival } \\
\hline & $R R(95 \% C I)$ & $\mathrm{P}$-value & $R R(95 \% C I)$ & $\mathrm{P}$-value \\
\hline \multicolumn{5}{|c|}{ All patients $(\mathrm{n}=300)$} \\
\hline RBM3 & \multicolumn{2}{|c|}{ Univariate } & \multicolumn{2}{|c|}{ Univariate } \\
\hline Low & 1.00 & & 1.00 & \\
\hline High & $0.70(0.50-0.99)$ & 0.04 & $0.61(0.42-0.87)$ & 0.006 \\
\hline RBM3 & \multicolumn{2}{|c|}{ Multivariate } & \multicolumn{2}{|c|}{ Multivariate } \\
\hline Low & 1.00 & & 1.00 & \\
\hline High & $0.78(0.51-1.19)$ & 0.25 & $0.68(0.44-1.06)$ & 0.09 \\
\hline RBM3 & \multicolumn{2}{|c|}{ Multivariate+treatment adjusted } & \multicolumn{2}{|c|}{ Multivariate+treatment adjusted } \\
\hline Low & 1.00 & & 1.00 & \\
\hline High & $0.79(0.51-1.20)$ & 0.27 & $0.69(0.44-1.07)$ & 0.10 \\
\hline \multicolumn{5}{|c|}{ ER positive $(\mathrm{n}=196)$} \\
\hline RBM3 & \multicolumn{2}{|c|}{ Univariate } & \multicolumn{2}{|c|}{ Univariate } \\
\hline Low & 1.00 & & 1.00 & \\
\hline High & $0.55(0.36-0.85)$ & 0.006 & $0.52(0.33-0.81)$ & 0.003 \\
\hline RBM3 & \multicolumn{2}{|c|}{ Multivariate } & \multicolumn{2}{|c|}{ Multivariate } \\
\hline Low & 1.00 & & 1.00 & \\
\hline High & $0.57(0.36-0.90)$ & 0.02 & $0.49(0.30-0.79)$ & 0.004 \\
\hline RBM3 & \multicolumn{2}{|c|}{ Multivariate+treatment adjusted } & \multicolumn{2}{|c|}{ Multivariate+treatment adjusted } \\
\hline Low & 1.00 & & 1.00 & \\
\hline High & $0.56(0.36-0.90)$ & 0.02 & $0.49(0.30-0.79)$ & 0.004 \\
\hline
\end{tabular}

Multivariate analysis included adjustment for grade, age, nodal status, ER status, PR status, Her2 and tumor size.

by altering microRNA levels. ${ }^{27}$ RBPs have also been described as a novel family of apoptosis modulators, ${ }^{1}$ and a correlation between the X chromosomerelated $R B M$ genes $(R B M X, R B M 3$ and $R B M 10)$ and the pro-apoptotic Bax gene has been shown in breast cancer. ${ }^{5}$ Our data add to these findings by the observation that increased RBM3 expression was associated with favorable clinicopathological variables and an improved prognosis in two independent breast cancer cohorts.

In vitro studies of the role of RBM3 in tumor progression have been conflicting. Baldi et $a l^{9}$ showed that $R B M 3$ was downregulated in metastatic melanoma cell lines, whereas Sureban et $a 1^{10}$ 
described $R B M 3$ as a proto-oncogene that prevents mitotic catastrophe in colorectal cancer cell lines. In the study by Sureban et al, ${ }^{10}$ immunohistochemistry was performed in a small number of colorectal cancer specimens. It is not clear which subcellular location was evaluated, and no relationship with prognosis was described.

Our data emphasize the fact that antibody-based approaches, using immunohistochemistry-based analysis of clinically annotated tumor specimens, are indispensable in the validation of putative cancer biomarkers. Such an approach allows for the assessment of candidate biomarker expression in different subcellular locations and their prognostic impact. We identified nuclear RBM3 as an independent prognostic marker (Tables 2 and 3); however, the ratio of nuclear to cytoplasmic staining may also affect disease outcome, eg, as previously shown for survivin in breast cancer using automated image analysis. ${ }^{28}$ It is noteworthy that, as visualized in Figures $3 a$ and $b$, the survival trends for the intermediate categories, ie, tumors with $1-25 \%$ nuclear positivity, were inconsistent between the two cohorts. However, the number of tumors in this group was rather small, particularly in cohort I $(10.8 \%)$. In cohort II, survival curves for RBM3negative and high tumors are clearly separated at 5 years of follow-up (Figure 3c), and then converging. In this study, we used the same scoring system as in the protein atlas for the extended analysis, but further studies are warranted to determine optimal cutoff values.

Breast cancer has revolutionized the application of biomarkers. Assessment of hormone receptor status and Her2 is compulsory for all newly diagnosed breast cancers. Nevertheless, additional biomarkers are required to identify prognostic subgroups and to tailor individual treatment regimens. ${ }^{29}$ The role of adjuvant chemotherapy in node-negative patients remains controversial. At present, the majority of premenopausal women with node-negative disease receive adjuvant chemotherapy; however, the absolute survival benefit from treatment is only $3 \%$ at 5 years and $5 \%$ at 10 years. ${ }^{30}$ It has been estimated that $70-80 \%$ of these patients, who are inherently at low risk, would have survived without adjuvant therapy and thus avoided potentially toxic side effects; however, there is a lack of accurate prognostic markers to identify those patients who would benefit from chemotherapy. ${ }^{31}$

Thus, an urgent need exists to identify markers, which would allow for the selection of a 'good prognosis' subgroup that may not require adjuvant systemic chemotherapy. The findings from cohort II indicated that the prognostic value of RBM3 seemed to be independent of tamoxifen treatment and $<2 \%$ of the patients received adjuvant chemotherapy. In light of this, RBM3 may be a promising marker for selecting patients who will not benefit from adjuvant treatment.
Taken together, our data show that high nuclear expression of RBM3 is clearly associated with a significantly improved survival, particularly in ERpositive tumors. Although some studies may contradict these findings, ${ }^{10}$ the majority of studies on members of the extended RBM family suggest a protective role in tumor progression. RBM8A and RBM8B interact with the tumor suppressor gene OVCA $1,{ }^{32}$ and RBMY is downregulated during testicular cancer progression. ${ }^{33,34}$ Additional studies on well-annotated tumor samples are warranted to confirm the utility of RBM3 as a prognostic biomarker in various cancer forms.

\section{Acknowledgements}

We thank Elise Nilsson for excellent assistance with construction of the tissue microarrays. This work was supported by grants from Swegene, the Knut and Alice Wallenberg Foundation, the Swedish Foundation for Strategic Research, the Swedish Cancer Society, Gunnar Nilsson's Cancer Foundation, the Crafoord Foundation and the Research funds of Malmö University Hospital. The UCD Conway Institute is funded by the Programme for Third Level Institutions (PRTLI), as administered by the Higher Education Authority (HEA) of Ireland.

\section{Disclosure/conflict of interest}

The authors declare no conflict of interest.

\section{References}

1 Sutherland LC, Rintala-Maki ND, White RD, et al. RNA binding motif (RBM) proteins: a novel family of apoptosis modulators? J Cell Biochem 2005;94:5-24.

2 Burd CG, Dreyfuss G. Conserved structures and diversity of functions of RNA-binding proteins. Science 1994;265:615-621.

3 Derry JM, Kerns JA, Francke U. RBM3, a novel human gene in Xp11.23 with a putative RNA-binding domain. Hum Mol Genet 1995;4:2307-2311.

4 Wright CF, Oswald BW, Dellis S. Vaccinia virus late transcription is activated in vitro by cellular heterogeneous nuclear ribonucleoproteins. J Biol Chem 2001;276:40680-40686.

5 Martinez-Arribas F, Agudo D, Pollan M, et al. Positive correlation between the expression of X-chromosome RBM genes (RBMX, RBM3, RBM10) and the proapoptotic Bax gene in human breast cancer. J Cell Biochem 2006;97:1275-1282.

6 Martin-Garabato E, Martinez-Arribas F, Pollan M, et al. The small variant of the apoptosis-associated $\mathrm{X}$-chromosome RBM10 gene is co-expressed with caspase-3 in breast cancer. Cancer Genomics Proteomics 2008;5:169-173.

7 Kita H, Carmichael J, Swartz J, et al. Modulation of polyglutamine-induced cell death by genes identified by expression profiling. Hum Mol Genet 2002;11: 2279-2287. 
8 Danno S, Nishiyama H, Higashitsuji H, et al. Increased transcript level of RBM3, a member of the glycine-rich RNA-binding protein family, in human cells in response to cold stress. Biochem Biophys Res Commun 1997;236:804-807.

9 Baldi A, Battista T, De Luca A, et al. Identification of genes down-regulated during melanoma progression: a cDNA array study. Exp Dermatol 2003;12:213-218.

10 Sureban SM, Ramalingam S, Natarajan G, et al. Translation regulatory factor RBM3 is a proto-oncogene that prevents mitotic catastrophe. Oncogene 2008;27:4544-4556.

11 Uhlen M, Bjorling E, Agaton C, et al. A human protein atlas for normal and cancer tissues based on antibody proteomics. Mol Cell Proteomics 2005;4: 1920-1932.

12 Ponten F, Jirstrom K, Uhlen M. The Human Protein Atlas-a tool for pathology. J Pathol 2008;216:387-393.

13 Kampf C, Andersson AC, Wester K, et al. Antibodybased tissue profiling as a tool for clinical proteomics. Clinical Proteomics 2004;1:285-299.

14 Andersson AC, Stromberg S, Backvall $\mathrm{H}$, et al. Analysis of protein expression in cell microarrays: a tool for antibody-based proteomics. J Histochem Cytochem 2006;54:1413-1423.

15 Borgquist S, Holm C, Stendahl M, et al. Oestrogen receptors alpha and beta show different associations to clinicopathological parameters and their co-expression might predict a better response to endocrine treatment in breast cancer. J Clin Pathol 2008;61:197-203.

16 Helczynska K, Larsson AM, Holmquist Mengelbier L, et al. Hypoxia-inducible factor-2alpha correlates to distant recurrence and poor outcome in invasive breast cancer. Cancer Res 2008;68:9212-9220.

17 Ryden L, Jonsson PE, Chebil G, et al. Two years of adjuvant tamoxifen in premenopausal patients with breast cancer: a randomised, controlled trial with longterm follow-up. Eur J Cancer 2005;41:256-264.

18 Rydén L, Stendahl M, Jonsson H, et al. Tumor-specific VEGF-A and VEGFR2 in postmenopausal breast cancer patients with long-term follow-up. Implication of a link between VEGF pathway and tamoxifen response. Breast Cancer Res Treat 2005;89:135-143.

19 Brennan DJ, Jirstrom K, Kronblad A, et al. CA IX is an independent prognostic marker in premenopausal breast cancer patients with one to three positive lymph nodes and a putative marker of radiation resistance. Clin Cancer Res 2006;12:6421-6431.

20 Kononen J, Bubendorf L, Kallioniemi A, et al. Tissue microarrays for high-throughput molecular profiling of tumor specimens. Nat Med 1998;4:844-847.

21 Nilsson P, Paavilainen L, Larsson K, et al. Towards a human proteome atlas: high-throughput generation of mono-specific antibodies for tissue profiling. Proteomics 2005;5:4327-4337.

22 Björling E, Lindskog C, Oksvold P, et al. A web-based tool for in silico biomarker discovery based on tissuespecific protein profiles in normal and cancer tissues. Mol Cell Proteomics 2007;7:825-844.

23 Lofstedt T, Jogi A, Sigvardsson M, et al. Induction of ID2 expression by hypoxia-inducible factor-1: a role in dedifferentiation of hypoxic neuroblastoma cells. J Biol Chem 2004;279:39223-39231.

24 Wellmann S, Buhrer C, Moderegger E, et al. Oxygenregulated expression of the RNA-binding proteins RBM3 and CIRP by a HIF-1-independent mechanism. J Cell Sci 2004;117:1785-1794.

25 Kitagawa K, Niikura Y. Caspase-independent mitotic death (CIMD). Cell Cycle 2008;7:1001-1005.

26 Saito Y, Nakagami H, Kurooka M, et al. Cold shock domain protein A represses angiogenesis and lymphangiogenesis via inhibition of serum response element. Oncogene 2008;27:1821-1833.

27 Dresios J, Aschrafi A, Owens GC, et al. Cold stressinduced protein Rbm3 binds $60 \mathrm{~S}$ ribosomal subunits, alters microRNA levels, and enhances global protein synthesis. Proc Natl Acad Sci USA 2005;102: 1865-1870.

28 Brennan DJ, Rexhepaj E, O’Brien SL, et al. Altered cytoplasmic-to-nuclear ratio of survivin is a prognostic indicator in breast cancer. Clin Cancer Res 2008;14: 2681-2689.

29 Brennan DJ, Kelly C, Rexhepaj E, et al. Contribution of DNA and tissue microarray technology to the identification and validation of biomarkers and personalised medicine in breast cancer. Cancer Genomics Proteomics 2007;4:3-16.

30 EBCTCG. Polychemotherapy for early breast cancer: an overview of the randomised trials. Lancet 1998;352: 930-942.

31 Brennan DJ, O’Brien SL, Fagan A, et al. Application of DNA microarray technology in determining breast cancer prognosis and therapeutic response. Expert Opin Biol Ther 2005;5:1069-1083.

32 Salicioni AM, Xi M, Vanderveer LA, et al. Identification and structural analysis of human RBM8A and RBM8B: two highly conserved RNA-binding motif proteins that interact with OVCA1, a candidate tumor suppressor. Genomics 2000;69:54-62.

33 Lifschitz-Mercer B, Elliott DJ, Leider-Trejo L, et al. Absence of RBM expression as a marker of intratubular (in situ) germ cell neoplasia of the testis. Hum Pathol 2000;31:1116-1120.

34 Schreiber L, Lifschitz-Mercer B, Paz G, et al. Lack of RBM expression as a marker for carcinoma in situ of prepubertal dysgenetic testis. J Androl 2003;24:78-84.

Supplementary Information accompanies the paper on Modern Pathology website (http://www.nature.com/ modpathol) 